Monatsschrift f. Geburtshülfe u. Gynäkologie 1936;102:256

\title{
Hochschul- und Tagesnachrichten
}

Münster. Dem Oberarzt der Frauenklinik Dr. med habil. Hermann Goecke wurde eine Dozentur für Geburtshilfe und Gynäkologie verliehen.

W i e n. Dr. Tassilo Antoine hat sich für Geburtshilfe und Gynäkologie habilitiert. - Dozent /. Amreich wurde zum ao. Professor für Geburtshilfe und Gynäkologie in Innsbruck ernannt. Die Nordostdeutsche Gesellschaft für Gynäkologie (Vorsitzender Prof. Dr. H. Fuchs, Danzig) stiftete zwei Ehrenpreise von je RM. 100,bzw. RM. 50,- für die beiden besten im SS. 1936 und WS. 1936/37 aus der Universität Königsberg i. Pr. und der Staatl. Akademie für praktische Medizin in Danzig hervorgehenden geburtshilflich-gynäkologischen Dissertationen. H. Fuchs. 